Rabelo ISR, Medeiros Filho AEC \& Pontes Jr JAF (2020). Training of the physical education professional to work in the children and adolescent health: an integrative review. Research, Society and Development, 9(7): 1-22, e558974369.

\title{
Formação do profissional de educação física para atuação na saúde da criança e do adolescente: uma revisão integrativa
}

Training of the physical education professional to work in the children and adolescent health: an integrative review

\section{Formación del profesional de educación física para trabajar en la salud de niños y adolescentes: una revisión integrativa}

Recebido: 09/05/2020 | Revisado: 12/05/2020 | Aceito: 18/05/2020 | Publicado: 30/05/2020

\section{Iriadelia Soraya Ribeiro Rabelo}

ORCID: https://orcid.org/0000-0002-0973-2262

Universidade Estadual do Ceará, Brasil

E-mail: soraribeiro@ hotmail.com

Antônio Evanildo Cardoso de Medeiros Filho

ORCID: https://orcid.org/0000-0002-4442-162X

Universidade Estadual do Ceará, Brasil

E-mail: evanildofilho17@gmail.com

José Airton de Freitas Pontes Junior

ORCID: https://orcid.org/0000-0003-2045-2461

Universidade Estadual do Ceará, Brasil

E-mail: jose.airton@uece.br

\section{Resumo}

Os professores de Educação Física podem desempenhar um papel fundamental na qualidade da saúde da criança e do adolescente, fazendo-se necessário uma formação inicial e continuada que proporcione conhecimentos teóricos e práticos para atender as demandas recorrentes nesse cenário de atuação. Considerando a relevância do assunto proposto, o estudo teve como objetivo averiguar a produção científica na área da Saúde da Criança e do Adolescente na formação de professores de Educação Física por meio de uma revisão integrativa da literatura. As buscas bibliográficas foram realizadas nas bases de dados eletrônicas LILACS, MEDLINE / PubMed, Web of Science e Scielo mediante combinações dos Descritores de Ciências da Saúde (DeCS). A partir da análise crítica dos artigos 
selecionados podemos destacar, dentre outros aspectos, que há implementação e aprimoramento de políticas públicas destinada ao público infantil (categoria 3) com o objetivo de melhorar a situação das crianças e adolescentes por meio da atuação do profissional de Educação Física. Dessa forma, é importante que os professores busquem estreitar as relações entre teoria e prática pedagógica, bem como inovar, ou seja, experimentar novos modelos, estratégias, metodologias e conteúdos, para que possam contribuir para a formação integral das crianças e adolescentes.

Palavras-chave: Formação de professores; Educação superior; Formação continuada; Desenvolvimento profissional.

\begin{abstract}
Physical Education teachers can play a fundamental role in the quality of child and adolescent health, making initial and continuing training necessary to provide theoretical and practical knowledge to meet the recurring demands in this scenario. In order to consider the relevance of the proposed subject, the study aimed to investigate the scientific production in the Child and Adolescent Health area in the training of Physical Education teachers through an integrative literature review. Bibliographic searches were performed in the electronic databases LILACS, MEDLINE / PubMed, Web of Science and Scielo using combinations of Health Sciences Descriptors (DeCS). From the critical analysis of the selected articles, it can be highlighted, among other aspects, that there are implementation and improvement of public policies aimed at children (category 3 ) with the aim of improving the situation of children and adolescents through the performance of the Physical Education professional. Thus, it is important that teachers seek to strengthen the relationship between theory and pedagogical practice, as well as innovating, which is, experimenting with new models, strategies, methodologies, and contents, so that they can contribute to the integral education of children and adolescents.
\end{abstract}

Keywords: Teacher training; Higher education; Continuing education; Professional development.

\title{
Resumen
}

Los docentes de educación física pueden desempeñar un papel fundamental en la calidad de la salud de los niños y adolescentes, haciendo que la capacitación inicial y continua sea necesaria para proporcionar conocimiento teórico y práctico para satisfacer las demandas 
recurrentes en este escenario. Considerando la relevancia del tema propuesto, el estudio tuvo como objetivo investigar la producción científica en el área de Salud Infantil y Adolescente en la capacitación de maestros de Educación Física a través de una revisión bibliográfica integradora. Se realizaron búsquedas bibliográficas en las bases de datos electrónicas LILACS, MEDLINE / PubMed, Web of Science y Scielo utilizando combinaciones de Descriptores de Ciencias de la Salud (DeCS). A partir del análisis crítico de los artículos seleccionados, podemos destacar, entre otros aspectos, que existe una implementación y mejora de las políticas públicas dirigidas a los niños (categoría 3) con el objetivo de mejorar la situación de los niños y adolescentes a través del desempeño del profesional de la Educación Física. Por lo tanto, es importante que los docentes busquen fortalecer la relación entre la teoría y la práctica pedagógica, así como innovar, es decir, experimentar con nuevos modelos, estrategias, metodologías y contenidos, para que puedan contribuir a la educación integral de niños y adolescentes.

Palabras clave: Formación docente; Educación universitária; Educación Continua; Desarrollo profesional.

\section{Introdução}

É indispensável à formação do profissional de Educação Física para atuar em seu ambiente de trabalho de acordo com as competências de sua profissão. As Diretrizes Curriculares Nacionais Para a Saúde (Brasil, 2008) nos cursos de graduação também discute a importância dessa formação, revelando que o currículo deve ser mais integrado e menos fragmentado, contribuindo, assim, para a formação de profissionais mais críticos, reflexivos fazendo com que os professores sejam capazes de criar situações e condições de aprendizagem nas quais o objetivo seja a construção de saberes a partir dos conhecimentos prévios frente às situações problemas.

De acordo com a Bendrath, Basei, \& Rodrigues (2017) a oferta da Educação Física de qualidade depende de educadores bem qualificados, as autoridades responsáveis devem realizar análises formativas dos sistemas de formação de professores de Educação Física de qualidade e considerá-las como uma prioridade política. Análises devem ser realizadas para melhoria tanto na formação inicial como na formação continuada de professores de Educação Física. 
A infância e a adolescência correspondem às fases mais importantes do desenvolvimento em relação aos aspectos motores. Isso ocorre, porque o organismo se encontra especialmente sensível à influência dos fatores ambientais, tanto de natureza positiva como negativa. Os autores descrevem ainda que o estabelecimento de índices de desempenho motor, nesses períodos, poderá contribuir decisivamente para a promoção da saúde coletiva.

Esta revisão integrativa justifica-se pela a necessidade de estudos que proporcionassem ferramentas para melhorar a qualidade na formação dos profissionais de Educação Física direcionada à saúde das crianças e adolescentes, portanto, o estudo teve como objetivo averiguar a produção científica na área da Saúde da Criança e do Adolescente na formação de professores de Educação Física por meio de uma revisão integrativa da literatura.

\section{Metodologia}

Este estudo se caracteriza como uma revisão integrativa, sendo esta uma abordagem metodológica que permite incluir estudos experimentais e não experimentais, permitindo uma compreensão integral do fenômeno estudado, combinando dados teóricos e empíricos da literatura. Incorpora outras possibilidades a que se destina, tais como: descrição de conceitos, revisão de ideias, ou hipóteses, análise minuciosa de problemas, permite uma larga amostra sincrônica de múltiplas propostas, gerando um panorama que consiste na compreensão de princípios complexos e eixos temáticos de maior relevância (Souza, Silva, \& Carvalho, 2010).

Considerando esse contexto e a relevância do assunto proposto, a revisão integrativa foi realizada a partir da busca de estudos acerca da formação em Educação Física relacionada à saúde de crianças e adolescentes. Esses estudos foram analisados para possibilitar uma compreensão sobre a realidade que cerca esse tema no cenário acadêmico.

As buscas foram realizadas nas bases de dados eletrônicas LILACS, MEDLINE / PubMed, Web of Science e Scielo, mediante combinações dos Descritores de Ciências da Saúde (DeCS) e palavras de texto foram usadas para gerar a lista de citações.

O modo de seleção dos estudos foi de acordo com Barbosa et al. (2012), no qual descreve a seguinte forma de seleção dos estudos: Os artigos foram selecionados de acordo com um método sistemático. Os critérios de inclusão para o estudo foram baseados em uma combinação de três parâmetros de busca: ensino, público-alvo e cenário; artigos escritos na língua portuguesa, inglesa ou espanhola; artigos que tenham como foco de análise a formação 
em Educação Física na intervenção em saúde de crianças e adolescentes; pesquisas realizadas no âmbito nacional e internacional; e artigos que se enquadrem no corte temporal de 15 anos (2004-2018).

Os critérios de exclusão utilizados foram: arquivos corrompidos com visualização indisponível; artigos que não tenham relação com o tema proposto; arquivos de acesso restrito.

Primeiramente os títulos e resumos dos artigos foram lidos, então os critérios de inclusão foram aplicados à análise. Todos os artigos de texto completo foram obtidos e incluídos se atendessem aos critérios de inclusão. As referências para todos os trabalhos selecionados foram examinadas para identificar outras publicações que devem ser revisadas.

As palavras-chave para o grupo ensino tiveram os seguintes descritores: "Formation" OR “Training” OR "Higher education”. O público-alvo (“Children” OR “Adolescents”) para localizar estudos potencialmente relevantes. O operador booleano "AND" foi usado para combinar os três grupos na pesquisa. O cenário utilizado teve "Physical education " como descritor.

Os descritores utilizados na busca estavam em conformidade com a terminologia dos Descritores em Ciências da Saúde (DeCS), as quais foram combinados para resultarem em artigos diretamente relacionados ao assunto. Portanto, foi utilizado o seguinte modelo de busca: Modelo de busca: \#1 AND \#2 AND \#3 ("Formation" OR "Training" OR "Higher education”) AND (“Children” OR “Adolescents”) AND ("Physical education").

É importante mencionar que a pesquisa seguiu as recomendações das normas estabelecidas na Resolução $n^{\circ}$ 510/16, nos termos do Art. 1², cláusula VI - "pesquisa realizada exclusivamente com textos científicos para revisão da literatura científica" (Brasil, 2016).

\section{Resultados e Discussão}

Com a equação foram encontrados nas seguintes bases de dados: LILACS ( $n=6350)$, MEDLINE / PubMed ( $\mathrm{n}=1928)$, Web of Science ( $\mathrm{n}=56.858)$ e Scielo ( $\mathrm{n}=271)$. Após seleção dos artigos pelo recorte temporal (2004-2018), texto completo e tipo de língua estrangeira, foram selecionados: LILACS ( $\mathrm{n}=2296)$ artigos, MEDLINE / PubMed ( $\mathrm{n}=351)$, Web of Science $(n=1668)$ e Scielo $(n=263)$. 
Research, Society and Development, v. 9, n. 7, e558974369, 2020

(CC BY 4.0) | ISSN 2525-3409 | DOI: http://dx.doi.org/10.33448/rsd-v9i7.4369

Após aplicação dos filtros 290 artigos atenderam aos critérios. Após a leitura atenta dos títulos e resumos bem-estruturados, 47 trabalhos atenderam aos critérios de inclusão e foram selecionados. Após leitura na íntegra desses artigos, 20 atenderam aos critérios de inclusão, os quais compuseram esta revisão integrativa. A Figura 1 mostra o diagrama da seleção dos artigos para a revisão integrativa.

Figura 1. Diagrama de seleção de artigos para revisão integrativa.

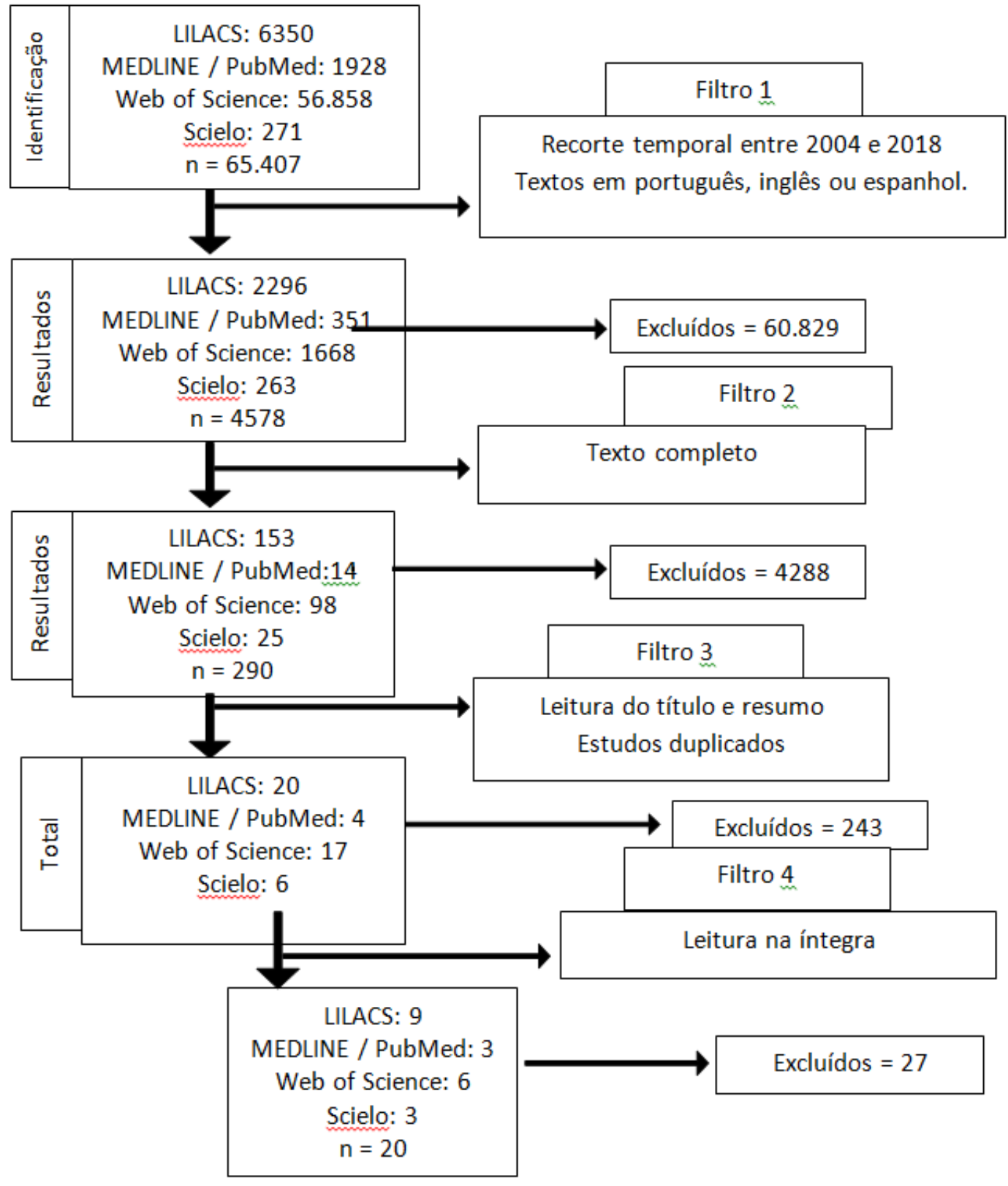

Fonte: Elaboração própria. 
Research, Society and Development, v. 9, n. 7, e558974369, 2020

(CC BY 4.0) | ISSN 2525-3409 | DOI: http://dx.doi.org/10.33448/rsd-v9i7.4369

A partir dos trabalhos selecionados mediante a leitura na íntegra, foi possível formular 3 categorias temáticas, sendo elas: i) Formação do profissional de Educação Física; ii) Educação Física e Saúde voltada para Crianças e Adolescentes, iii) Políticas Públicas Infantis.

Os estudos selecionados serão apresentados seguindo as respectivas categorias temáticas formuladas, com destaque para os principais resultados e conclusões encontrados nos artigos analisados. Nas Tabelas a seguir podem ser encontradas características dos artigos selecionados para revisão baseada em autores/ano, título do estudo e principais resultados. Desse modo, a na Tabela 1 podem ser consultados as características dos estudos referentes à categoria "formação do profissional de Educação Física". 
Tabela 1. Categoria I: Formação do profissional de Educação Física.

\begin{tabular}{|c|c|}
\hline AUTORES/ ANO & $\begin{array}{l}\text { PRINCIPAIS RESULTADOS } \\
\end{array}$ \\
\hline $\begin{array}{l}\text { Cardoso, Dos Reis, } \\
\& \text { Iervolino (2008) }\end{array}$ & $\begin{array}{l}\text { Necessidade imediata de um processo de educação permanente, para } \\
\text { que os professores conseguissem ampliar seus conceitos de saúde e } \\
\text { trabalharem com os princípios da Escola Promotora de Saúde, em prol } \\
\text { da qualidade de vida de todos aqueles que convivem no ambiente } \\
\text { escolar. }\end{array}$ \\
\hline $\begin{array}{l}\text { Hayes (2008) } \\
\text { Web of Science }\end{array}$ & $\begin{array}{l}\text { O conhecimento de conteúdo foi visto como tendo maior importância } \\
\text { para professores e mentores de estudantes, mas tutores universitários } \\
\text { geralmente conceituaram o conhecimento do assunto de forma mais } \\
\text { ampla, sugerindo que ele deveria ser visto como abrangendo uma série } \\
\text { de bases de conhecimento necessárias para o ensino. }\end{array}$ \\
\hline $\begin{array}{l}\text { Neves (2012) } \\
\text { Web of Science }\end{array}$ & $\begin{array}{l}\text { Foi observada uma articulação entre os programas de pós-graduação, } \\
\text { os cursos de formação e as escolas da rede pública de educação básica. }\end{array}$ \\
\hline $\begin{array}{l}\text { Constantinides } \\
\quad(2013)\end{array}$ & $\begin{array}{l}\text { Especialistas em Educação Física exibiram níveis de ensino mais } \\
\text { eficazes nas aulas práticas de Educação Física do que os professores } \\
\text { não especialistas. }\end{array}$ \\
\hline Web of Science & \\
\hline $\begin{array}{c}\text { Carvalho, Prado, \& } \\
\text { Alonso (2013) } \\
\text { Web of Science } \\
\end{array}$ & $\begin{array}{l}\text { A aproximação entre estudantes, profissionais e usuários do serviço de } \\
\text { saúde. }\end{array}$ \\
\hline $\begin{array}{l}\text { García, Fernández, } \\
\text { Prete, \& Olivares } \\
\text { (2014) } \\
\text { Web of Science }\end{array}$ & $\begin{array}{l}\text { Grandes diferenças encontradas no autoconceito físico de acordo com } \\
\text { o nível socioeconômico, mas não de acordo com o gênero. Os } \\
\text { resultados sugerem uma mudança na metodologia de ensino dos } \\
\text { professores de Educação Física. }\end{array}$ \\
\hline $\begin{array}{l}\text { Meás, Amaya, } \\
\text { Rada, \& García } \\
\text { (2014) } \\
\text { SCIELO }\end{array}$ & $\begin{array}{l}\text { Coordenação entre o sistema de saúde e o sistema educacional, } \\
\text { satisfazendo e estimulando a intervenção criativa tanto para o grupo } \\
\text { escolar quanto para os profissionais de saúde como participantes. } \\
\text { Observou-se a educação em saúde do próprio estudo com a demanda } \\
\text { dos alunos, satisfazendo e estimulando a intervenção criativa tanto para } \\
\text { o grupo escolar como para os profissionais de saúde como } \\
\text { participantes. }\end{array}$ \\
\hline $\begin{array}{l}\text { Dyrstad, Kvalo, } \\
\text { Alstveit, \& Skage } \\
\text { (2018) } \\
\text { LILACS }\end{array}$ & $\begin{array}{l}\text { Lições fisicamente ativas } \\
\text { Facilidade de organizar lições. Inclusão de lições fisicamente ativas } \\
\text { nos currículos. Recepção positiva das crianças da intervenção. }\end{array}$ \\
\hline
\end{tabular}

Fonte: Elaboração própria.

A primeira categoria refere-se à formação do profissional de Educação Física. Foram analisados oito estudos relacionados a esse tema (Cardoso, Dos Reis, \& Iervolino, 2008; Hayes, 2008; Neves, 2012; Constantinides, 2013; Carvalho, Prado, \& Alonso, 2013; García et al., 2014; Meás, Amaya, Rada, \& García, 2014; Dyrstad, et al., 2018).

O estudo de Cardoso, Dos Reis, e Iervolino (2008) teve como objetivo identificar os conhecimentos dos professores em relação aos temas de saúde e promoção da saúde, bem 
como verificar o interesse e a necessidade dos professores em capacitações nos temas relacionados à saúde e educação e saúde.

Na visão das autoras, estes professores deveriam ser capacitados para a observação da saúde, desta forma eles conseguiriam perceber a criança em todas as suas necessidades e não só suas doenças. Seria preciso entender as relações que elas têm com o mundo em que vivem, com suas famílias e grupo social a que pertencem.

Ao término deste estudo verificou-se que não foi possível propor um processo de educação continuada, para que as escolas se tornassem promotoras de saúde, entretanto foi observado o conhecimento, interesse e necessidade que os professores têm em capacitações de temas relacionados à saúde e educação em saúde.

O estudo de Hayes (2008) foi realizado com professores universitários que trabalhavam em três cursos de formação inicial de professores de Educação Física na Inglaterra. $\mathrm{O}$ objetivo era que o conhecimento fosse priorizado por professores, alunos e mentores escolares. O conhecimento de conteúdo foi visto como tendo maior importância para professores e mentores de estudantes, mas tutores universitários geralmente conceituam o conhecimento do assunto de forma mais ampla, sugerindo que ele deveria ser visto como abrangendo uma série de bases de conhecimento necessárias para o ensino.

Diante dessa perspectiva, cabe salientar que o professor exerce uma influência constante e ativa sobre os conceitos de saúde e doença dos seus alunos, sua sensibilidade e didática devem dar condições de repassar informações acerca de saúde e adaptá-las ao ambiente escolar, necessitando assim de um suporte na área para subsidiar o seu trabalho. Geralmente o professor se torna referência para os alunos e pode estimular a compreensão e adoção de hábitos saudáveis, além disso, um professor preparado para observar corretamente o ambiente escolar e perceber os riscos pode proteger a saúde dos escolares e seus familiares (Oliveira, 2001).

O estudo de Neves (2012) relatou que com a publicação da Lei $\mathrm{n}^{\circ} 11.502$ de 2007, a Capes recebeu as atribuições de induzir e fomentar a formação inicial e continuada de profissionais da educação básica, além de estimular a valorização do magistério em todos os níveis e modalidades de ensino. Os resultados mostraram a titulação de doutores e mestres e a formação de graduandos e professores da rede pública em torno de projetos de cursos, estudos e pesquisas, promovendo, assim, o diálogo entre o universo da escola e o da educação superior, criando e inovando para transformar. 
Sabe-se da importância da formação do profissional de Educação Física para atuar em seu ambiente de trabalho de acordo com as competências de sua profissão. As Diretrizes Curriculares Nacionais Para a Saúde (DCNS, 2008) nos cursos de graduação também discute a importância dessa formação, revelando que o currículo deve ser mais integrado e menos fragmentado, contribuindo, assim, para a formação de profissionais mais críticos, reflexivos fazendo com que os professores sejam capazes de criar situações e condições de aprendizagem nas quais o objetivo seja a construção de saberes a partir dos conhecimentos prévios frente às situações problemas.

O estudo de Constantine (2013) teve como objetivo comparar os processos de ensino nas aulas de Educação Física, ministradas por especialistas e não especialistas. Especialistas em Educação Física exibiram níveis de ensino mais eficazes. Enquanto os professores especialistas ofereciam diversas atividades e testes em suas aulas práticas para a aquisição das habilidades, os "não especialistas" forneciam apenas o jogo e menos oportunidades práticas.

Quanto ao estudo de Carvalho (2013), teve como proposta apresentar como o campo denominado saúde coletiva/saúde pública e as políticas/os programas de saúde, em particular o PET-Saúde, e debater se eles têm respondido aos dilemas da formação em saúde, especialmente na atenção primária à saúde/atenção básica.

Os resultados encontrados indicaram que as metodologias de ensino devem ser revisadas e ampliadas, de modo a incluir outras formas de ensino, pois não se aprende apenas por transmissão cognitiva. Para incorporação de saberes e práticas, são necessárias as experiências, as exposições de si e o contato com a alteridade (o outro despertando a diferença em nós). A implicação dos estudantes revelou-se determinante no seu protagonismo, a ser reconhecido e incentivado.

O estudo de Dyrstad, et al. (2018) na Noruega descreveu que para melhorar a saúde e a aprendizagem acadêmica em crianças em idade escolar, foi utilizado o programa Active School em Stavanger, que introduzia lições acadêmicas fisicamente ativas, um método de ensino que combinava atividade física com conteúdo acadêmico. Este método foi bem recebido entre os líderes escolares, professores e crianças, sendo aceitos como uma forma significativa de aumentar tanto o aprendizado quanto a atividade física e a saúde embora a grande maioria de professores preferiu que houvesse a redução de tempo de atividade física.

A aprovação da política nacional de promoção da saúde, em março de 2006, afirma que a promoção da saúde deve ser entendida como uma estratégia para fomentar a qualidade 
de vida, e auxiliar na redução das vulnerabilidades e riscos relacionados aos determinantes e condicionantes da saúde da população (Brasil, 2006).

De acordo com Yoon \& Armour (2017), a aprendizagem profissional dos professores de Educação Física e os impactos sobre o aprendizado de alunos em uma comunidade na Coréia do Sul após a implantação de um novo currículo nacional de Educação Física, no qual os professores foram desafiados a entregar novos resultados educacionais com o objetivo de compartilhar a aprendizagem e impactar em pedagogias, práticas e aprendizado de alunos em desenvolvimento de caráter por meio da Educação Física.

Hildebrandt-Stramann (2008), em seu estudo sobre formação de professores de Educação Física para escolas públicas na Alemanha, abordou uma formação moderna de professores com dois objetivos gerais: qualificar os estudantes para realizar um ensino de Educação Física que cumpra exigências pedagógicas modernas; e participar de uma configuração escolar em que o movimento seja uma parte integral da formação escolar e, com isso, do processo de reflexão e conscientização sobre o desenvolvimento infantil.

De acordo com a Política Nacional de Promoção à Saúde (PNPS), a promoção, a informação e a educação em Saúde devem ter ênfase na promoção de atividade física, buscando consolidar a pesquisa de saúde dos escolares como forma de monitoramento de práticas corporais e atividade física de adolescentes (Brasil, 2006).

O problema dos graduados de ensino superior tem um significado especial, em particular dos especialistas em treinamento físico e esporte. Esses especialistas são os portadores e tradutores de valores de cultura física e geral e transmitem conhecimentos e habilidades práticas para cultura educacional e física, atividade esportiva, saúde, modo de vida, e tempo de lazer, contribuindo assim para a melhoria de cultura social.

É por isso que pode ter relação com o aumento do nível de cultura profissional pedagógica do futuro especialista na esfera da cultura física e do esporte é um problema real no espaço educacional moderno (Ivanii, 2016). Diante do exposto, pode-se perceber que os estudos atuais estão se direcionando para uma nova política de formação do profissional de Educação Física. 
Tabela 2. Categoria II: Educação Física e Saúde voltada para Crianças e Adolescentes.

\begin{tabular}{|c|c|}
\hline AUTORES/ ANO & $\begin{array}{l}\text { PRINCIPAIS RESULTADOS } \\
\end{array}$ \\
\hline $\begin{array}{l}\text { Nakamura et al. } \\
\text { (2013) }\end{array}$ & $\begin{array}{l}\text { O presente estudo verificou que é possível que as aulas de Educação } \\
\text { Física desenvolvidas dentro da escola, possam aumentar o nível total de } \\
\text { Atividade Física, principalmente para os meninos. Os resultados } \\
\text { também mostraram que os adolescentes que não participaram de } \\
\text { atividades esportivas apresentaram menos chances de serem ativos do } \\
\text { que aqueles que praticaram alguma. }\end{array}$ \\
\hline $\begin{array}{l}\text { Bergmann et al. } \\
\qquad(2013) \\
\text { MEDLINE / } \\
\text { PubMed }\end{array}$ & $\begin{array}{l}\text { A prevalência de adolescentes inativos foi elevada. Estratégias de } \\
\text { combate à inatividade física em adolescentes devem ser elaboradas } \\
\text { tendo a escola e a família papel de destaque. }\end{array}$ \\
\hline $\begin{array}{l}\text { Barbosa Filho } \\
\text { (2014) } \\
\text { LILACS }\end{array}$ & $\begin{array}{l}\text { Foi observado uma prevalência de inatividade física, comportamentos } \\
\text { sedentários e hábitos alimentares pouco saudáveis }\end{array}$ \\
\hline $\begin{array}{l}\text { Moraes et al. } \\
\quad(2014) \\
\text { Web of Science }\end{array}$ & $\begin{array}{l}\text { Trabalhar de forma integrada para contemplar as demandas dos } \\
\text { usuários, respeitando os limites de cada área do conhecimento sem } \\
\text { sobreposição das mesmas, buscando superar a formação fragmentada } \\
\text { dos docentes e acadêmicos. }\end{array}$ \\
\hline $\begin{array}{l}\text { Sporh et al. } \\
\text { (2014) } \\
\text { LILACS } \\
\end{array}$ & $\begin{array}{l}\text { Aumento de conhecimento em saúde entre os alunos em curto espaço de } \\
\text { tempo }\end{array}$ \\
\hline $\begin{array}{l}\text { Sanches Junior, } \\
\text { Carvalho, Salerno, } \\
\& \text { Araújo (2015) } \\
\text { LILACS }\end{array}$ & $\begin{array}{l}\text { Visão dos gestores em estarem contribuindo com a inclusão enquanto os } \\
\text { professores se mostrando com sentimentos de incapacidade para } \\
\text { implantá-la nesse momento. }\end{array}$ \\
\hline
\end{tabular}

Fonte: Elaboração própria.

A segunda categoria corresponde a Educação Física e saúde voltada para crianças e adolescentes. Foram analisados seis trabalhos sobre a temática (Nakamura et al., 2013; Bergmann et al., 2013; Barbosa Filho, 2014; Moraes et al., 2014; Spohr et al., 2014; Sanches Junior, Carvalho, Salerno, \& Araújo, 2015).

O estudo de Nakamura et al. (2013) foi realizado em escolas privadas e públicas da Rede de Ensino Médio de Rio Claro, no Estado de São Paulo e consistia em medir o nível de Atividade Física durante o tempo de lazer dos adolescentes. O estudo contou com a participação de 15 escolas (10 públicas e 5 privadas). Os principais resultados foram que as meninas tiveram menor prevalência de Atividade Física total que os meninos, e os meninos que praticaram Educação Física e atividades esportivas dentro da escola foram mais propensos a serem ativos no lazer do que os adolescentes que não praticaram tais atividades. 
A pesquisa de Bergmann (2013) foi realizado com escolares de 10 a 17 anos de idade, matriculados durante o período diurno, nos sistemas municipal e estadual de ensino, em Uruguaiana (RS). Os resultados do estudo indicaram que mais de dois terços da amostra foram classificados como fisicamente inativos. Esse procedimento, além de identificar os fatores associados à inatividade física em adolescentes de 10 a 17 anos, pode contribuir para a elaboração de programas de prevenção da inatividade física e para promover a adesão ao exercício físico regular.

Vários estudos, como o de Costa (2014), analisaram a influência da família nos níveis de atividade física em crianças e adolescentes e que o apoio dos pais possui influência na prática de atividade física de seus filhos, sendo a atividade física uma medida profilática contra as doenças crônicas não transmissíveis e o comportamento sedentário está associado ao aumento de doenças crônicas não transmissíveis.

O estudo de Barbosa Filho (2012) foi uma revisão integrativa sobre epidemiologia da inatividade física, comportamentos sedentários e hábitos alimentares não saudáveis em adolescentes brasileiros. Os estudos analisados indicaram um crescente interesse em pesquisas epidemiológicas relacionadas à atividade física/inatividade entre adolescentes. Esta revisão forneceu um quadro indicando que a inatividade física afeta pelo menos a metade dos adolescentes em diversos locais do Brasil. Esta revisão demonstra que a maioria dos estudos encontrou uma prevalência de comportamento sedentário.

Considerando que as DNTs são as principais causas de morte em adultos brasileiros, se políticas públicas de combate a esses fatores comportamentais de risco para DCNT não forem desenvolvidas, estimativas futuras podem ser ainda mais preocupantes.

Por sua vez, Moraes et al. (2014) apresentou um relato qualitativo sobre as atividades de um projeto do qual participaram docentes e acadêmicos dos cursos de Educação Física, Enfermagem, Nutrição e Odontologia. Os autores apresentaram diferentes reflexões quanto à abordagem da atenção à saúde de crianças e adolescentes. Além disso, o autor ressaltou que os investimentos financeiros e políticas públicas são relevantes para manutenção e intensificação de projetos com propostas de mudanças na formação profissional, pois refletem na qualidade de vida dos usuários de serviços de saúde.

Mostra-se relevante caracterizar o estudo de Spohr et al. (2014) que teve como objetivo foi avaliar a efetividade do projeto "Educação Física +: Praticando Saúde na Escola" $(\mathrm{EF}+)$, desenvolvido a partir das aulas de Educação Física no ensino fundamental e médio da rede pública da cidade de Pelotas/RS. No projeto, 40 escolas foram sorteadas e alocadas nos 
grupos controle e intervenção, utilizando estratégias como: oficina de formação de professores; entrega de material didático aos professores; e entrega de cartazes ilustrativos. No total, 4418 alunos e 19 professores participaram da coleta de dados nos dois períodos. Os autores concluíram que a proposta era exequível e contribuiu para o aumento de conhecimento em saúde entre os alunos em curto espaço de tempo.

Segundo o Estatuto da Criança e do Adolescente (1990), é dever da família, da comunidade, da sociedade em geral e do poder público assegurar, com absoluta prioridade, a efetivação dos direitos referentes à saúde, à educação, ao esporte e ao lazer das crianças e adolescentes. Portanto, crianças e adolescentes têm direito à proteção à vida e à saúde, mediante a efetivação de políticas sociais públicas que permitam o nascimento e o desenvolvimento sadio e harmonioso, em condições dignas de existência.

Corroborando, Freire (2016) menciona que promover a atividade física na infância e na adolescência estabelece uma base sólida para redução da prevalência do sedentarismo na idade adulta, o que contribui para uma melhor qualidade de vida.

É notável uma preocupação com as políticas de Educação Física relacionada a crianças e adolescentes, pois a compreensão aprimorada do impacto dos determinantes sociais na saúde infantil e nos resultados do desenvolvimento ressalta a importância de um quadro em evolução para a transformação do sistema com implicações políticas importantes (Dworkin, 2016). Na Tabela 3, pode ser consultados os principais apontamentos dos estudos referente à categoria temática "Políticas Públicas Infantis". 
Tabela 3. Categoria III: Políticas Públicas Infantis.

\begin{tabular}{cl}
\hline $\begin{array}{c}\text { AUTORES/ } \\
\text { ANO }\end{array}$ & \multicolumn{1}{c}{ PRINCIPAIS RESULTADOS } \\
\hline $\begin{array}{c}\text { Evenson, Ballard, } \\
\text { \& Ammerman } \\
\text { (2009). }\end{array}$ & $\begin{array}{l}\text { Com a implementação da política teve aumento do foco dos alunos nos } \\
\text { estudos, participação na atividade física, conscientização sobre hábitos } \\
\text { saudáveis, alerta e prazer e maior envolvimento da equipe. Produzindo, } \\
\text { assim muitos resultados positivos para estudantes e funcionários }\end{array}$ \\
$\begin{array}{c}\text { MEDLINE / } \\
\text { PubMed }\end{array}$ & $\begin{array}{l}\text { Produção de guias técnicos, boas práticas locais e projetos-piloto } \\
\text { realizados pelo setor acadêmico, em parceria com Prefeitos e iniciativa } \\
\text { privada, fornecendo abordagens e metodologias de intervenção } \\
\text { específicas que devem ser tomadas em conta para o redesenho a Política }\end{array}$ \\
$\begin{array}{c}\text { Salinas \& Rio } \\
\text { (2011) } \\
\text { de Promoção da Saúde Escolar, que as novas autoridades governamentais }\end{array}$ \\
SCIELO realizar.
\end{tabular}

Nobre et al. 2014

Atrasos nas habilidades motoras fundamentais foram detectados em estudantes em situações de risco. 3,4 Esse fenômeno sugere a necessidade de que essa informação seja levantada, levando em consideração o (LILACS) contexto e o gênero da criança para o desenvolvimento de políticas públicas mais efetivas. As aulas de Educação Física são elaboradas por professores de sala de aula que se sentem despreparados e comprometem o desenvolvimento motor das crianças.

Lonsdale, et al. (2016)

Este projeto fornecerá evidências e uma estrutura para o governo orientar a promoção da atividade física em todas as escolas primárias de NSW e LILACS) um modelo potencial para adoção em outros estados e países. Aumento os salários dos professores

Fonte: Elaboração própria.

Portanto, a terceira categoria compreende políticas públicas infantis, e corresponde a seis artigos (Evenson, Ballard, \& Ammerman, 2009; Salinas \& Rio, 2011; Alves, Chaves, \& Gontijo, 2012; Thompson, Linchey, \& Madsen, 2013; Nobre et al., 2014; Lonsdale et al., $2016)$.

O estudo de Evenson, Ballard, \& Ammerman (2009) descreveu como os municípios estão cumprindo o tempo relacionado à atividade física de uma política de saúde para crianças saudáveis. Essa política de saúde exigiu que todas as crianças, desde os jardins de infância até 
a oitava série recebessem pelo menos 30 minutos de moderada a vigorosa atividade física por dia. Os resultados demonstraram numerosos efeitos positivos da política nas escolas primárias e médias.

Quantos aos benefícios podem ser mencionados o aumento do foco do aluno em estudos, participação na atividade física, conscientização sobre hábitos saudáveis, alerta e prazer e maior envolvimento dos alunos. Os desafios de implementação para a política incluíram falta de tempo na rotina da escola e falta de participação dos professores.

Dessa maneira, destaca-se a importância da criação e manutenção de políticas específicas para as crianças e adolescentes com foco desde a formação do profissional que vai acompanha-las em todo seu processo de desenvolvimento.

No estudo de Salinas e Rio (2011) temos estratégias e ações realizadas, principalmente pela união dos setores acadêmicos e privados, resultando principalmente em modelos de intervenção e materiais técnicos de educação nutricional sendo necessário criar uma instituição nacional responsável pela implementação de uma política pública coerente que incorpore tanto a academia quanto o setor privado na política de Promoção de Saúde Escolar. Os principais resultados deste estudo mostram que há maior conscientização do público sobre o tema Promoção da Saúde Escolar, conscientização e criação de redes de trabalho locais.

Por sua vez, o estudo de Alves, Chaves, e Gontijo (2012) discute a integração do educador físico na rede de suporte social de crianças e adolescentes em situação de vulnerabilidade, discutindo em seu texto que a Educação Física tem sido cada vez mais convidada para compor equipes em programas sociais possibilitando, assim a reflexão sobre a importância de os cursos formadores no Ensino Superior oferecerem especial atenção à realização de interfaces com outras áreas do conhecimento, facilitando a compreensão do profissional em relação ao seu papel junto à rede de proteção dos direitos da criança e do adolescente.

A partir da compreensão do esporte como um fenômeno social relevante no contexto social brasileiro, a gestão pública deve, ao propor programas sociais, promover adequada capacitação dos educadores na promoção de ativa reflexão e mudança da práxis, gerando, assim, intervenções que fortaleçam a rede de suporte social.

Além disso, a inclusão de educadores sociais na rede de atenção aos direitos da criança e do adolescente deve ser efetivada a partir de políticas que garantam a qualidade do atendimento, integrando cada programa e profissional a uma efetiva rede de atenção. 
O estudo Nobre et al. (2014) revela em seu corpo textual que as políticas públicas para intercâmbio social não favorecem as crianças com menos habilidades motoras e que as únicas opções de vida eram os projetos sociais. Diz ainda que nas escolas pesquisadas as aulas de Educação Física ficavam a critério dos gestores escolares, e quando havia eram apenas uma vez por semana. O estudo observacional revelou que o estilo de vida das crianças estava alinhado com as oportunidades ofertadas no contexto, sustentando a hipótese de que contextos com características similares às que foram identificadas neste estudo apresentaram grande probabilidade de terem crianças com atrasos motores.

Conforme Bendrath, Basei, \& Rodrigues (2017), a oferta da Educação Física de qualidade depende de educadores bem qualificados. Dessa forma, as autoridades responsáveis devem realizar análises pontuais dos sistemas de formação de professores de Educação Física de qualidade e considerá-las como uma prioridade política. As análises devem ser realizadas para melhorias tanto na formação inicial como na formação continuada de professores de Educação Física.

Visto isso, a Educação Física e seus professores precisam fundamentar-se teoricamente para justificar a comunidade escolar e à própria sociedade o que já sabem fazer. Além disso, os professores devem estreitar as relações entre teoria e prática pedagógica, bem como inovar, ou seja, experimentar novos modelos, estratégias, metodologias e conteúdos, para que a Educação Física contribua para a formação integral das crianças e jovens e para a apropriação crítica da cultura contemporânea.

\section{Considerações Finais}

A partir dos resultados podemos argumentar que o campo da formação dos profissionais de Educação Física na intervenção na saúde de crianças e adolescentes vem sendo discutido por outros pesquisadores com foco na formação do profissional de Educação Física (categoria 1) e na preocupação com a Educação Física e saúde voltada para crianças e adolescentes (categoria 2).

Destaca-se, todavia, considerações importantes encontradas nos artigos sobre a construção e mudanças em políticas públicas infantis (categoria 3) com o objetivo de melhorar a situação das crianças e adolescentes por meio da atuação dos profissionais de Educação Física no âmbito escolar. 
Diante disso, os professores precisam estreitar as relações entre teoria e prática pedagógica, bem como inovar, ou seja, experimentar novos modelos, estratégias, metodologias e conteúdos, para que a Educação Física contribua para a formação integral das crianças e adolescentes. A partir desse estudo espera-se contribuir para uma melhor qualidade na formação dos professores de Educação Física direcionada a saúde das crianças e adolescentes.

\section{Referências}

Alves, H. C., Chaves, A. D., \& Gontijo, D. T. (2012). “uma andorinha só não faz verão”: a integração do educador físico na rede de suporte social de crianças e adolescentes em situação de vulnerabilidade: discussões a partir de um curso de educação continuada. Pensar $a$ Prática, 15(2). Doi: https://doi.org/10.5216/rpp.v15i2.12772.

Barbosa Filho, V. C., Campos, W. D., \& Lopes, A. D. S. (2012). Prevalence of alcohol and tobacco use among Brazilian adolescents: a systematic review. Revista de saude publica, 46(5), 901-917. Doi: https://doi.org/10.1590/S0034-89102012000500018

Barbosa Filho, V. C., Campos, W. D., \& Lopes, A. D. S. (2014). Epidemiology of physical inactivity, sedentary behaviors, and unhealthy eating habits among brazilian adolescents a systematic review. Ciência \& saúde coletiva, 19(1), 173-194. Doi:

https://doi.org/10.1590/1413-81232014191.0446.

Bendrath, Basei, \& Rodrigues, F. S. (2017). UNESCO. Diretrizes em Educação Física de qualidade: para gestores de políticas. Brasília: UNESCO, 2015. Práxis Educativa, Ponta Grossa, 12(2), 591-594, 2017. Doi: 10.5212/PraxEduc.v.12i2.0017.

Bergmann, G. G., Bergmann, M. L. D. A., Marques, A. C., \& Hallal, P. C. (2013). Prevalence of physical inactivity and associated factors among adolescents from public schools in Uruguaiana, Rio Grande do Sul State, Brazil. Cadernos de saude publica, 29, 2217-2229. Doi: https://doi.org/10.1590/0102-311x00077512. 
BRASIL. Ministério da Educação e Cultura Diretrizes Curriculares Nacionais para os cursos da Saúde. Brasília, DF, 2008.

Brasil. (2006). Ministério da Saúde. Política Nacional de Promoção da Saúde. Brasília: MS.

Brasil. (1990). Lei $n^{o}$ 8069, de 13 de julho de 1990. Dispõe sobre o Estatuto da Criança e do Adolescente e dá outras providências.

Cardoso, V., Dos Reis, A. P., Iervolino, S. A. (2008) Escolas promotoras de saúde. Journal of Human Growth and Development, v.18, n.2, p.107-115. DOI:

https://doi.org/10.7322/jhgd.19872.

Carvalho, Y. M., Prado, A. R., \& Alonso, A. T. (2013). Formação em Educação Física no Brasil: outros modos de pensar e intervir no serviço público de saúde. Educación Física y Ciencia, 15(1). Recuperado de <http://sedici.unlp.edu.ar/handle/10915/33651>

Neves, C. M. (2012). A Capes e a formação de professores para a educação básica. Revista Brasileira de Pós-Graduação, supl. 2, p353-373.

Constantinides, P., Montalvo, R., \& Silverman, S. (2013). Teaching processes in elementary physical education classes taught by specialists and nonspecialists. Teaching and Teacher Education, 36, 68-76. DOI: 10.1016/j.tate.2013.07.004.

Costa, T. R. A. (2014). Apoio social e atividade física em crianças e adolescentes: uma revisão da literatura. 22 f. Monografia (Especialização) - Curso de Fisiologia do Exercício, Universidade Federal do Paraná, Curitiba.

Dyrstad, S. M., Kvalo, S. E., Alstveit, M., \& Skage, I. (2018). Physically active academic lessons: acceptance, barriers and facilitators for implementation. BMC public health, 18(1), 322. Recuperado de <https://bmcpublichealth.biomedcentral.com/articles/10.1186/s12889018-5205-3>. 
Dworkin, P. H., \& Sood, A. B. (2016). A Population Health Approach to System Transformation for Children's Healthy Development. Child and Adolescent Psychiatric Clinics, 25(2), 307-317. Doi: 10.1371/journal.pone.0218403.

Evenson, K. R., Ballard, K., Lee, G., \& Ammerman, A. (2009). Implementation of a school-based state policy to increase physical activity. Journal of School Health, 79(5), 231238. Doi: 10.1111/j.1746-1561.2009.00403.x.

Freire, J. C., Moura, J. L. S., Nogueira, D. S., Souza, J. D., \& Cavalcanti, P. R. (2016). A influência familiar para a prática de exercícios físicos durante a infância e suas consequências na vida adulta de estudantes de educação física. In Congresso Internacional de Atividade Física, Nutrição e Saúde (No. 1). Recuperado de <https://eventos.set.edu.br /index.php/CIAFIS/article/view/3104>.

García, J., Fernández, M., Del Prete, A., \& Olivares, P. R. (2014). Autoconcepto físico de escolares chilenos. Diferencias en función del género y del estatus socioeconómico y su relación con la intensidad percibida en la clase de educación física. Motricidad Humana, 15(2), 63-71. Disponível em: $\langle$ https://dialnet.unirioja.es/servlet/articulo?codigo=6348115>.

Oliveira, D. G. (2001) O professor alfabetizador como multiplicador de saúde. Periódico online. Recuperado de <http://www.unoesc.br/site/comunidade/comprojetos2.asp> .

Meás, F. G., Amaya, A. J. R., Rada, L. P. V. D., \& García, J. M. D. (2014). Intervención comunitaria en centro educativo: entrevista a un profesional sanitario. Ene, 8(2). Doi: http://dx.doi.org/10.4321/S1988-348X2014000200005.

Hayes, S., Capel, S., Katene, W., \& Cook, P. (2008). An examination of knowledge prioritisation in secondary physical education teacher education courses. Teaching and teacher education, 24(2), 330-342. Doi: https://doi.org/10.1016/j.tate.2006.10.012. 
Hildebrandt-Stramann, R. (2008). A formação de professores de educação física para escolas públicas na Alemanha. Journal of Physical Education, 14(2), 97-103. Recuperado de em:〈http://www.periodicos.uem.br/ojs/index.php/RevEducFis/article/view/3475/2489>.

Ivanii, I. (2016). Approaches of the professional-pedagogical culture to form students of physical culture specialties during the process of professional training. Journal of Physical Education and Sport, 16, 640. Recuperado de <https://www.questia.com/library/journal/1P34208088051/approaches-of-the-professional-pedagogical-culture>.

Lonsdale, C. et al. (2016). Scaling-up an efficacious school-based physical activity intervention: study protocol for the 'Internet-based Professional Learning to help teachers support Activity in Youth'(iPLAY) cluster randomized controlled trial and scale-up implementation evaluation. BMC public health, 16(1), 873. Doi: 10.1186/s12889-016-3243-2.

Moraes, R. B. et al. (2014). Ações multidisciplinares na saúde integrando ensino-serviço: uma experiência de dez anos. Cinergis, 15(3). DOI: http://dx.doi.org/10.17058/cinergis.v15i3.4917

Nakamura, P. M. et al. (2013). Physical education in schools, sport activity and total physical activity in adolescents. Revista Brasileira de Cineantropometria \& Desempenho Humano, 15(5), 517-526. Doi: https://doi.org/10.5007/1980-0037.2013v15n5p517.

Nobre, S., Salviano, F., Cambruzzi Coutinho, M. T., \& Valentini, N. C. (2014). A ecologia do desenvolvimento motor de escolares litorâneos do nordeste do brasil. Revista Brasileira de Crescimento e Desenvolvimento Humano, 24(3). Recuperado de <http://pepsic.bvsalud.org /scielo.php?script=sci_arttext\&pid=S0104-12822014000300005\&lng=pt\&nrm=iso\&tlng=pt $>$

Salinas, J., \& Rio, F. V. D. (2011). Programas de salud y nutrición sin política de Estado: el caso de la Promoción de Salud Escolar en Chile. Revista chilena de nutrición, 38(2), 100-116. Doi: http://dx.doi.org/10.4067/S0717-75182011000200001.

Sanches Junior, M. L., Carvalho, C. L., Salerno, M. B., \& Araújo, P. F. (2015). Concepções e práticas da inclusão na educação física escolar: estudo em uma cidade do Brasil. Educ. fis. deporte, 34(1) 155-179. DOI: 10.17533/udea.efyd.v34n1a07 
Souza, M. T. D., Silva, M. D. D., \& Carvalho, R. D. (2010). Revisão integrativa: o que é e como fazer. Einstein (São Paulo), 8(1), 102-106. Doi: https://doi.org/10.1590/s167945082010 rw1134.

Spohr, C. F., Fortes, M. O., Rombaldi, A. J., Hallal, P. C., \& Azevedo, M. R. (2014). Atividade física e saúde na Educação Física escolar: efetividade de um ano do projeto "Educação Física+". Revista Brasileira de Atividade Física \& Saúde, 19(3), 300-300. Doi: http://dx.doi.org/10.12820/rbafs.v.19n3p300

Thompson, H. R., Linchey, J., \& Madsen, K. A. (2013). Peer reviewed: are physical education policies working? A snapshot from San Francisco, 2011. Preventing chronic disease, 10. Doi: 10.5888/pcd10.130108.

Yoon, K., \& Armour, K. M. (2017). Mapping physical education teachers' professional learning and impacts on pupil learning in a community of practice in South Korea. Physical Education and Sport Pedagogy, 22(4), 427-444. Doi: 10.1080/17408989.2016.1268589.

\section{Porcentagem de contribuição de cada autor no manuscrito}

Iriadelia Soraya Ribeiro Rabelo - 50\%

Antonio Evanildo Cardoso de Medeiros Filho - 25\%

José Airton de Freitas Pontes Junior - 25\% 